\title{
Dysregulated STAT1-SOCS1 control of JAK2 promotes mammary luminal progenitor cell survival and drives $E R \alpha^{+}$tumorigenesis
}

\author{
SR Chan ${ }^{*, 1,4}$, CG Rickert ${ }^{1,4}$, W Vermi ${ }^{1,2}$, KCF Sheehan ${ }^{1}$, C Arthur ${ }^{1}$, JA Allen ${ }^{1}$, JM White ${ }^{1}$, J Archambault ${ }^{1}$, S Lonardi ${ }^{2}$, TM McDevitt ${ }^{3}$, \\ D Bhattacharya ${ }^{1}$, MV Lorenzi ${ }^{3,5}$, DC Allred ${ }^{1}$ and RD Schreiber ${ }^{*, 1}$
}

We previously reported that STAT1 expression is frequently abrogated in human estrogen receptor- $\alpha$-positive $\left(E R \alpha^{+}\right)$breast cancers and mice lacking STAT1 spontaneously develop ER $\alpha^{+}$mammary tumors. However, the precise mechanism by which STAT1 suppresses mammary gland tumorigenesis has not been fully elucidated. Here we show that STAT1-deficient mammary epithelial cells (MECs) display persistent prolactin receptor (PrIR) signaling, resulting in activation of JAK2, STAT3 and STAT5A/ $5 \mathrm{~B}$, expansion of $\mathrm{CD} 61^{+}$luminal progenitor cells and development of $\mathrm{ER} \alpha^{+}$mammary tumors. A failure to upregulate SOCS1, a STAT1-induced inhibitor of JAK2, leads to unopposed oncogenic PrIR signaling in STAT1 ${ }^{-I-}$ MECs. Prophylactic use of a pharmacological JAK2 inhibitor restrains the proportion of luminal progenitors and prevents disease induction. Systemic inhibition of activated JAK2 induces tumor cell death and produces therapeutic regression of pre-existing endocrine-sensitive and refractory mammary tumors. Thus, STAT1 suppresses tumor formation in mammary glands by preventing the natural developmental function of a growth factor signaling pathway from becoming pro-oncogenic. In addition, targeted inhibition of JAK2 may have significant therapeutic potential in controlling ER $\alpha^{+}$breast cancer in humans.

Cell Death and Differentiation (2014) 21, 234-246; doi:10.1038/cdd.2013.116; published online 13 September 2013

Estrogen receptor- $\alpha$-positive $\left(\mathrm{ER} \alpha^{+}\right)$breast cancer, the most commonly diagnosed subtype of breast malignancy in women, is routinely treated with combinations of endocrine therapies and radiation or chemotherapy. Nevertheless, half of the patients do not benefit from these treatments because of intrinsic or acquired resistance. ${ }^{1}$ Therefore, alternative therapeutic targets in $\mathrm{ER} \alpha^{+}$breast cancer need to be developed.

Work by others has revealed a mammary tumor-promoting effect of prolactin receptor (PrIR) signaling in humans and mice. A meta-analysis of epidemiological data concluded that women in the top quartile for serum prolactin (Prl) concentration have a $60 \%$ increased risk of developing $\mathrm{ER} \alpha^{+}$, but not $\mathrm{ER} \alpha^{-}$, breast cancer, compared with those in the bottom quartile. $^{2}$ Consistent with these findings, upregulated PrIR expression and constitutive activation of STAT3 and STAT5 is frequently found in human $\mathrm{ER} \alpha^{+}$breast cancers. ${ }^{3-6}$ These results suggest that PrIR signaling is uniquely associated with $\mathrm{ER} \alpha^{+}$breast cancer in humans. Animal studies also support this conclusion. Mice engineered for persistent PrIR signaling display significantly increased incidences of $\mathrm{ER} \alpha^{+}$mammary tumors. ${ }^{7,8}$ However, the natural processes that govern the pro-oncogenic potential of PrIR signaling remain largely unknown.

Under physiologic conditions, canonical PrIR signaling is initiated by Prl-mediated conformational change of the intracellular domains within two dimerized PrIR molecules, leading to activation of receptor-associated JAK2 and phosphorylation and activation of the transcription factors STAT5A and STAT5B (hereafter referred to as STAT5A/5B). ${ }^{9}$ Mice lacking STAT5A or engineered for selective loss of JAK2 in the mammary gland display the same mammary gland defects as $\mathrm{PrIR}^{-1-}$ mice, ${ }^{10-13}$ thus revealing that these signaling components mediate PrIR's action on mammary gland development. Importantly, ligand-induced activation of PrIR also causes phosphorylation of other STAT family members, notably STAT1 and STAT3. ${ }^{14-17}$ However, the physiological significance of STAT1 and STAT3 activation remains unclear.

We recently reported that STAT1-deficient mice spontaneously develop $\mathrm{ER} \alpha^{+}$mammary tumors that exhibit histopathological, biological, and genetic parallels to human $\mathrm{ER} \alpha^{+}$

\footnotetext{
${ }^{1}$ Department of Pathology and Immunology, Washington University School of Medicine, 425S Euclid Avenue, St. Louis, MO 63110, USA; ${ }^{2}$ Department of Molecular and Translational Medicine, Section of Pathology, University of Brescia School of Medicine, Piazzale Spedali Civili 1, Brescia 25123 , Italy and ${ }^{3}$ Oncology Drug Discovery, Research and Development, Bristol-Myers Squibb, Princeton, NJ 08543, USA

${ }^{*}$ Corresponding authors: SR Chan, Department of Pathology and Immunology, Campus Box 8118, Washington University School of Medicine, $425 S$ Euclid Avenue, St. Louis, MO 63110, USA. Tel: +314 362 8128; Fax: +314 747 0809; E-mail: schan@ pathology.wustl.edu

or RD Schreiber, Department of Pathology and Immunology, Campus Box 8118, Washington University School of Medicine, 425S Euclid Avenue, St. Louis, MO, 63110, USA. Tel: +314 362 8747; Fax: +314 747 0809; E-mail: schreiber@ immunology.wustl.edu

${ }^{4}$ These authors contributed equally to this work.

${ }^{5}$ Present address: Oncology Research and Development, Janssen Pharmaceutical Companies, Johnson and Johnson, Spring House, Pennsylvania 19477, USA Keywords: breast cancer; STAT1; SOCS1; ER $\alpha$; PrIR; JAK2

Abbreviations: $\mathrm{ER} \alpha$, estrogen receptor- $\alpha$; MEC, mammary epithelial cell; MIN, mammary intraepithelial neoplasia; OVX, ovariectomy; Prl, prolactin; PrIR, prolactin receptor; WT, wild type

Received 03.2.13; revised 23.7.13; accepted 24.7.13; Edited by RA Knight; published online 13.9.2013
} 
breast cancers. ${ }^{18} \mathrm{We}$ also found that STAT1 expression is significantly suppressed in the neoplastic cells of a large proportion of human $\mathrm{ER} \alpha^{+}$breast cancers, suggesting that downregulation of STAT1 is a critical step during tumorigenesis. ${ }^{18}$ Along with others, we further demonstrated that the tumor-suppressor function of STAT1 is epithelial intrinsic. ${ }^{18-21}$ However, the precise mechanism by which STAT1 suppresses mammary tumorigenesis has not been fully elucidated. Here we demonstrate that dysregulated PrIR-JAK2-STAT3/5A/5B activation promotes survival of mammary epithelial cells (MECs) and initiates development of $\mathrm{ER} \alpha^{+}$mammary tumors, and that STAT1 antagonizes this tumorigenic process by controlling JAK2 activation.

\section{Results}

Persistent PrIR-JAK2-STAT3/STAT5A/5B signaling in STAT1 $^{-I-}$ mammary tumors. STAT $1^{-/}$females are highly susceptible to the development of $\mathrm{ER} \alpha^{+}$mammary adenocarcinomas. ${ }^{18,19}$ These tumors develop at higher incidence following multiple pregnancies compared with nulliparous animals, ${ }^{18}$ suggesting that pregnancy-associated hormones such as estrogen, progesterone or Prl may have critical roles in the tumorigenic process. To investigate whether estrogen and progesterone are required to promote tumor progression, tumor-bearing STAT1 ${ }^{-1}$ mice were ovariectomized (OVX; Figure 1a). Although tumors continued to progress in sham-operated mice, tumors in the OVX mice regressed, albeit to different extents. Thirty-six percent $(5 / 14)$ of the tumors partially regressed, but then grew progressively while $64 \%(9 / 14)$ of the tumors regressed to undetectable levels with all recurring at the same sites. These results indicate that ovarian hormones are not essential in maintaining the tumorigenic phenotype of STAT1 ${ }^{-/-}$mammary tumors.

Given that human $\mathrm{ER} \alpha^{+}$breast cancer cells frequently display persistent PrIR signaling, ${ }^{3,4}$ we tested the alternative hypothesis that STAT1 ${ }^{-1}$ mammary tumors rely on PrIR signaling for continuous growth. We found elevated PrIR expression on primary cells from mammary intraepithelial neoplasia (MIN) and invasive carcinomas compared with nontransformed MECs, and on STAT1 ${ }^{-1-}$ $\mathrm{ER} \alpha^{+}$mammary tumor cell lines, SSM2 and SSM3 (ref. 18) (Figures $1 \mathrm{~b}$ and $\mathrm{c}$ ). In addition, JAK2 was persistently phosphorylated on key tyrosine residues (Y1007 and $\mathrm{Y} 1008)$ within the kinase domain in primary STAT1 ${ }^{-1-}$ mammary tumors and SSM2 and SSM3 tumor cell lines, but not in unstimulated MECs (NMuMG) nor in control $\mathrm{STAT}^{-1-}$ ER $\alpha$-negative SSM1 tumor cells ${ }^{18}$ (Figures 1d and e). This observation suggested that JAK2 was enzymatically active in STAT1 ${ }^{-1}-\mathrm{ER}^{+}$tumor cells. Indeed, these cells displayed tyrosine phosphorylated forms of STAT3 (pSTAT3) and STAT5A/5B (pSTAT5A/5B) (Figures 1d and e, Supplementary Figures S1B-S1C). STAT3/5A/5B activation was selective as STAT6 was not phosphorylated, and the tumor cells did not express STAT4 (Supplementary Figure S1D). When compared with normal mammary tissues, primary STAT1 ${ }^{-1}$ - mammary tumors overexpressed SOCS3 and SOCS2, which are target genes for pSTAT3 and pSTAT5A/5B, respectively (Figure 1f). Taken together, these results show that the signaling components constituting the PrIR pathway are activated in $\mathrm{STAT}^{-1-} \mathrm{ER}^{+}$mammary tumors.

PrIR and JAK2 are required for STAT3 and STAT5A/5B activation. We next used a loss-of-function approach to explore whether a cause-and-effect relationship existed between activation of PrIR, JAK2 and STAT3/5A/5B. PrIRspecific shRNAs were transduced into SSM2 and SSM3 cells, and caused a 40-60\% reduction in the amount of tyrosine phosphorylated JAK2 (pJAK2) and a 63-87\% reduction in pSTAT3 (Figures $2 A$ and $B$ and Supplementary Figures S2A and S2B). Thus, JAK2 and STAT3 activation is dependent on PrIR in STAT1 ${ }^{-1-}$ mammary tumor cells. Knockdown of JAK2 expression using JAK2-specific shRNAs reduced pSTAT3 levels by $70-80 \%$ and pSTAT5A/5B levels by $80-90 \%$ (Figures $2 \mathrm{c}$ and $\mathrm{d}$ and Supplementary Figure $\mathrm{S} 2 \mathrm{C}$ ). Inhibition of JAK2 activity by a small molecule JAK2selective inhibitor, BMS-911543, ${ }^{22}$ also caused a significant decrease in pSTAT3 and pSTAT5A/5B levels (Figure 2e), demonstrating that JAK2's kinase activity is required to activate both STAT3 and STAT5A/5B. Together, these results show that PrIR initiates the signaling cascade required for the subsequent activation of JAK2 that phosphorylates STAT3 and STAT5A/5B in STAT1 ${ }^{-/}$- mammary tumor cells.

\section{Persistent activation of PrIR-JAK2-STAT3/5A/5B provides} a critical survival signal. Constitutive activation of STAT3 has been observed in a wide variety of malignant cells, including breast cancer cells, and provides a survival signal that supports the transformed phenotype. ${ }^{23,24} \mathrm{We}$ observed that knockdown of PrIR significantly increased the percentages of apoptotic SSM2 and SSM3 cells (50-60\%) compared with cells treated with a control luciferase-specific shRNA (5-15\%; Figure 3a). Consistent with these results, knockdown of JAK2 also induced a 10- and 5-fold increase in apoptosis in SSM2 and SSM3, respectively, over control shRNAs (Figure 3b). Furthermore, pharmacological inhibition of JAK2 using BMS-911543 caused apoptosis in SSM2 and SSM3 cells (Figure 3c). If STAT3 and STAT5A/5B are the transcription factors that affect survival from PrIR-JAK2 signaling, blockade of their function should also induce tumor cell apoptosis. Indeed, introduction of a validated STAT3 binding-site decoy into SSM2 and SSM3 that abrogates induction of STAT3 target genes ${ }^{25}$ resulted in a three- to fourfold increase in apoptosis compared with the mutant form of the oligonucleotide (Figure 3d). STAT5A/5B was equally essential in maintaining tumor cell viability because shRNA-mediated knockdown of STAT5A/B induced a three- to fourfold increase in the percentages of early apoptotic cells in SSM2 and SSM3 over control shRNA (Figure 3e). In contrast, perturbation of PrIR, JAK2, STAT3 or STAT5A/B in control NMuMG or ER $\alpha$-negative SSM1 cells did not affect cellular viability (Figure 3 ). Taken together, these results show that STAT1-1- $\mathrm{ER} \alpha^{+}$ mammary tumor cells are addicted to PrIR-JAK2-STAT3/ $5 \mathrm{~A} / 5 \mathrm{~B}$ signaling for survival in vitro. 


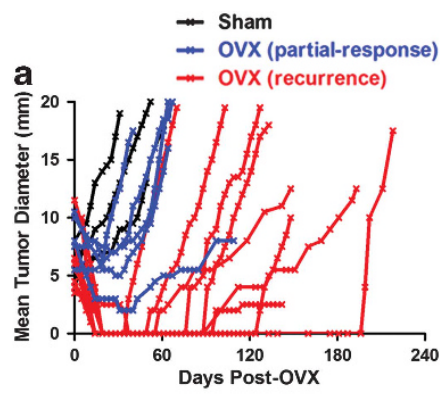

b
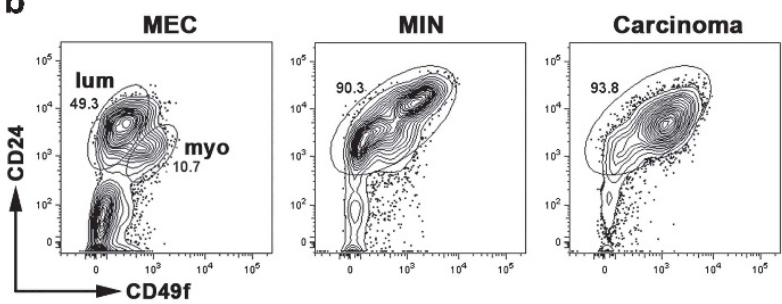

C
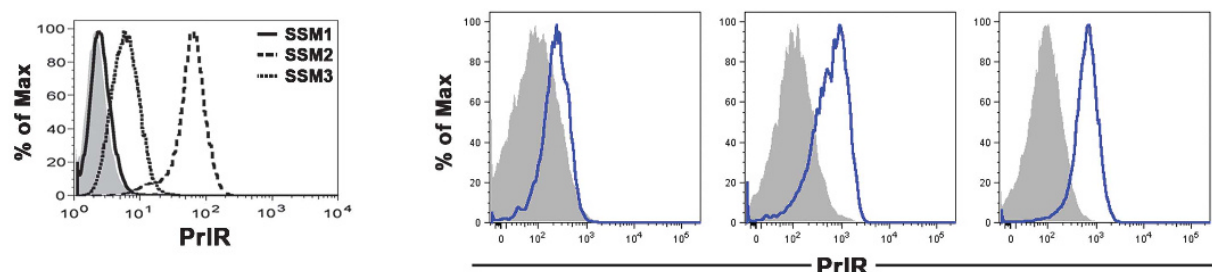

d

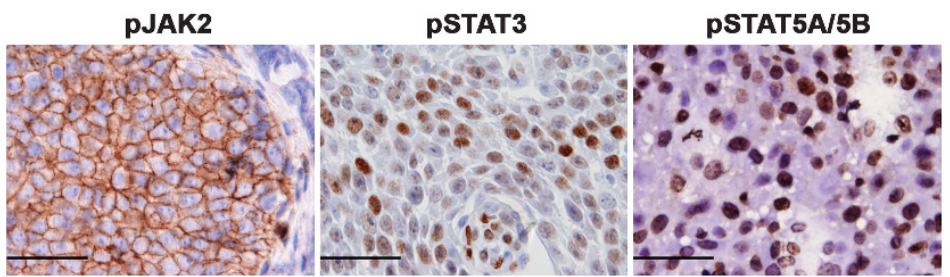

e



f

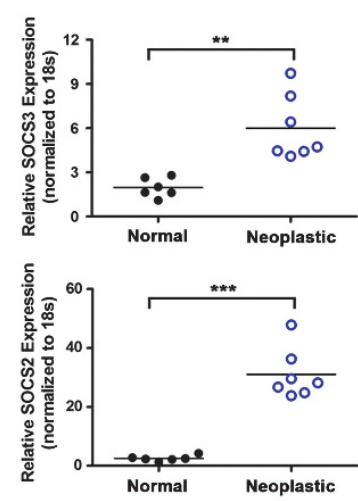

Figure 1 STAT1 ${ }^{-1-}$ mammary tumor cells exhibit persistent activation of JAK2, STAT3 and STAT5A/5B. (a) STAT1 ${ }^{-1-}$ mice bearing primary mammary tumors were sham-operated (black) or OVX (blue and red). Each line represents an individual tumor. (b) Disaggregated MECs and neoplastic cells from MIN (the earliest morphologically identifiable lesions) and carcinomas were gated based on CD49f and CD24 levels (top; circles). PrIR expression on CD49f ${ }^{\text {int }}$ CD24 ${ }^{\text {hi }}$ luminal (lum) epithelial cells was quantified using b-Prl and SA-PE (bottom). Representative flow cytometry plots from three independent STAT1 ${ }^{-1-}$ mice are shown. (c) ER $\alpha^{+}$ SSM2 and SSM3 cells displayed elevated PrIR expression compared with the control ER $\alpha^{-}$SSM1 cells. (d) Phosphorylated JAK2, STAT3 and STAT5 were observed in primary STAT1 ${ }^{-1-}$ mammary tumor cells using immunohistochemical analyses. Representative images of five primary mammary tumors are shown. Scale $\mathrm{bar}=33 \mu \mathrm{m}$. (e) Immunoprecipitation western blotting analysis of JAK1, JAK2, STAT3 and STAT5 in NMuMG, SSM1, SSM2 and SSM3 cells. IFN $\gamma$-stimulated NMuMG was used as a positive control. (f) SOCS3 and SOCS2 mRNA levels were quantified in nontransformed STAT1 ${ }^{-1-}$ mammary glands (normal) and STAT1 ${ }^{-1-}$ mammary tumors (neoplastic). ${ }^{\star \star} P<0.001,{ }^{* \star *} P<0.0001$

\section{Systemic inhibition of PrIR-JAK2-STAT3/5A/5B signaling causes regression of $\mathrm{STAT}^{-I-}$ mammary tumors.} To determine whether persistent activation of PrIR signaling was critical for promoting tumor cell survival in vivo, $\mathrm{STAT}^{-/-}$mice bearing primary mammary tumors were treated with vehicle or BMS-911543. BMS-911543 displayed a potent inhibitory effect on tumor cell signaling, as evidenced by the dramatic reduction in pSTAT3 and pSTAT5A/5B levels in the tumor cells within hours after the first dose of BMS-911543 was administrated (Figures $4 a$ and $b$ and Supplementary Figures S3A-S3D). Massive tumor cell death and infiltration of immune cells were observed in BMS-911543-treated, but not vehicletreated, animals. Interestingly, tumor cell proliferation was not affected by BMS-911543 (Figure 4a). After 3 weeks of treatment, tumors in all of the BMS-911543-treated mice regressed to undetectable size, whereas tumors in the control group continued to grow (Figures $4 c$ and $d$ ). Tumor 
a

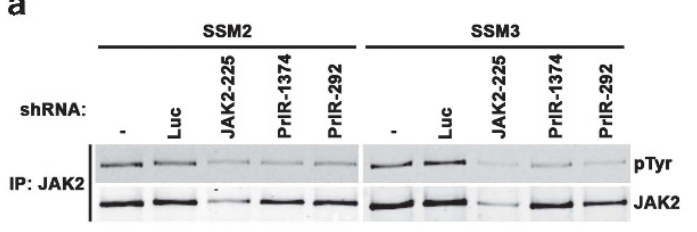

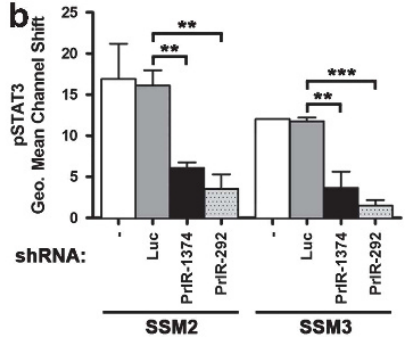
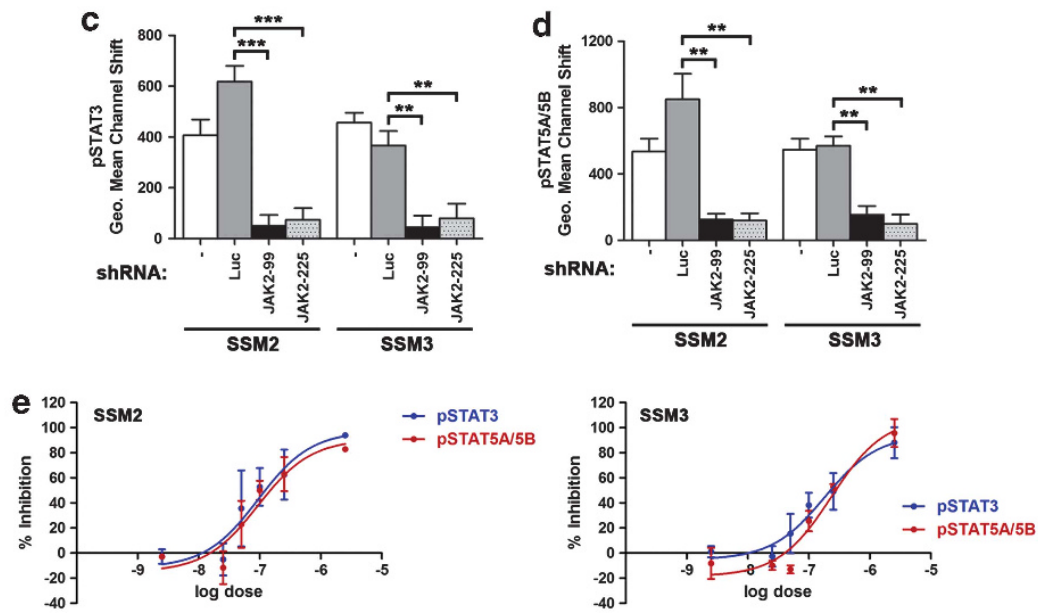

Figure 2 PrIR and JAK2 are required for activation of STAT3 and STAT5A/5B in STAT1 ${ }^{-1-}$ mammary tumor cells. (a) PrIR knockdown resulted in a reduction in pJAK2 levels in SSM2 and SSM3. Cells were also treated with a JAK2-specific shRNA (JAK2-225) as positive controls. (b) Knocking down PrIR led to a reduction in pSTAT3 in SSM2 and SSM3, as measured by flow cytometry. (c and d) Reduction in JAK2 expression significantly reduced the levels of pSTAT3 (c) and pSTAT5A/5B (d) in SSM2 and SSM3, as measured by flow cytometry. (e) Flow cytometry analysis of pSTAT3 and pSTAT5 in SSM2 and SSM3 cells treated with increasing doses of BMS-911543. IC $50=94$ nM for SSM2 and $182-234 \mathrm{nM}$ for SSM3. ${ }^{* \star} P<0.01,{ }^{* \star *} P<0.0001$. Error bars, S.E.M.

regression was durable since BMS-911543-treated mice followed up to 7 months after termination of treatment remained tumor free (Supplementary Figure S3E). Progression of transplanted SSM3 tumors was also blocked by BMS-911543 (Supplementary Figure S3F). These results show that $\mathrm{STAT}^{-/-} \mathrm{ER} \alpha^{+}$mammary tumor cells are addicted to PrIR-JAK2-STAT3/5A/5B signaling in vivo, and JAK2 inhibition results in the complete elimination of these tumors.

JAK2 activation confers ovarian hormone independency. As ovarian hormone depletion did not prevent outgrowth of $\mathrm{ER}^{+}{ }^{+}$mammary tumors (Figure 1a), but JAK2 inhibition did (Figure 4), we asked whether activated JAK2 had a causal role in steroid hormone-independent outgrowth. OVX mice bearing $\mathrm{ER} \alpha^{+}$hormone-independent tumors treated with BMS-911543 displayed nearly complete blockade of tumor growth while vehicle-treated mice showed continued tumor progression (Figure 4e and Supplementary Figures S3G-S3I). Thus, persistently activated JAK2 is a key driver of progression of both hormone-dependent and independent STAT1 ${ }^{-1-}$ mammary tumors.

Persistent JAK2 activation causes an expansion of luminal progenitor cells and initiates tumor development. We tested whether activated JAK2 was also involved in the initiation of mammary tumor development. STAT3 was uniquely activated in MECs of aged STAT1 ${ }^{-1-}$ mice before detectable malignancy, but not in age-matched wild-type (WT) controls (Figure 5a). In contrast, pSTAT5A/5B was observed in both WT and STAT1 ${ }^{-1-}$ mammary glands. Whole-mount analysis of mammary fat pads indicated a significant increase in the number of alveolar buds in STAT1 ${ }^{-1-}$ mice compared with WT mice (Figures $5 \mathrm{~b}$ and c). Excessive bud formation was observed in 8- to 12-month-old nulliparous and multiparous STAT1 ${ }^{-1}$ mice but was rare in young post-pubertal females of 3 months of age (data not shown). The aberrant appearance of the alveolar buds was not due to differences in serum levels of estradiol, progesterone, or Prl between WT and STAT1 ${ }^{-/-}$mice, or in the local production of Prl because Prl mRNA cannot be detected in STAT1 ${ }^{-1-}$ MECs (Supplementary Figure S4A and data not shown). In contrast, abnormal bud development was due to aberrant JAK2 activation because BMS-911543 markedly reduced the number of alveolar buds in STAT1 ${ }^{-/-}$ mammary glands (Figures $5 \mathrm{~b}$ and $\mathrm{c}$ ).

During murine mammary gland development, stem cells residing in the myoepithelial compartment differentiate into lineage-specific progenitor cells, which subsequently give rise to mature myoepithelial or luminal epithelial cells. ${ }^{26-28}$ As $\mathrm{STAT}^{-1}$ - mammary glands display over-exuberant development of alveolar buds, we examined whether STAT1 ${ }^{-1-}$ mammary glands contained an increased percentage of luminal progenitor cells compared with WT glands. Indeed, 


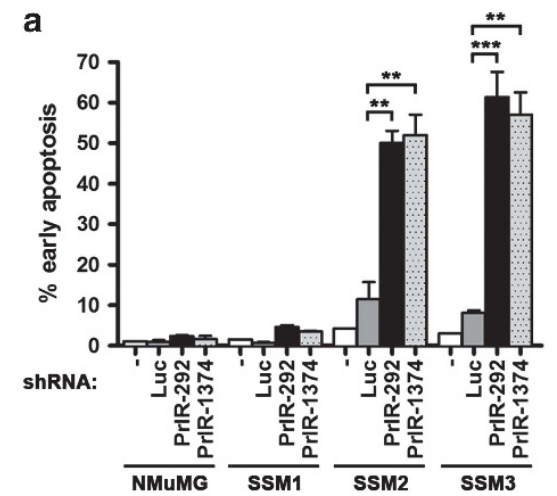

b
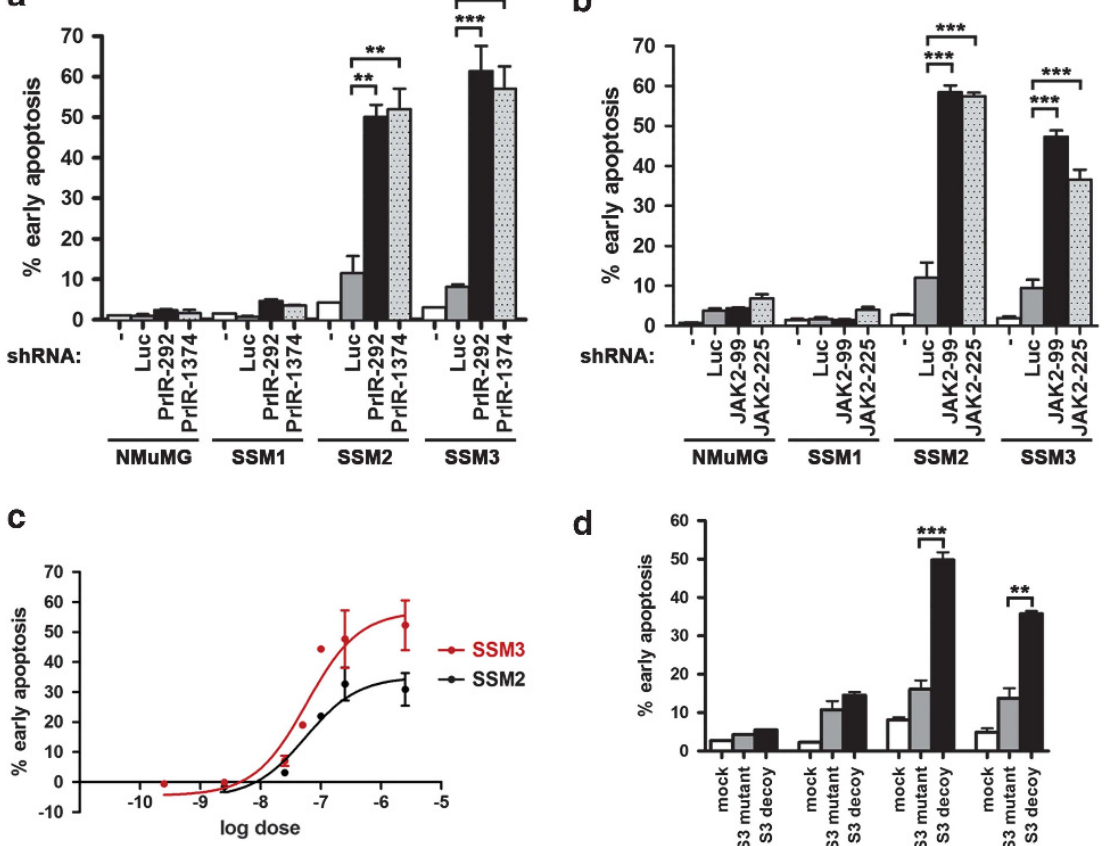

d
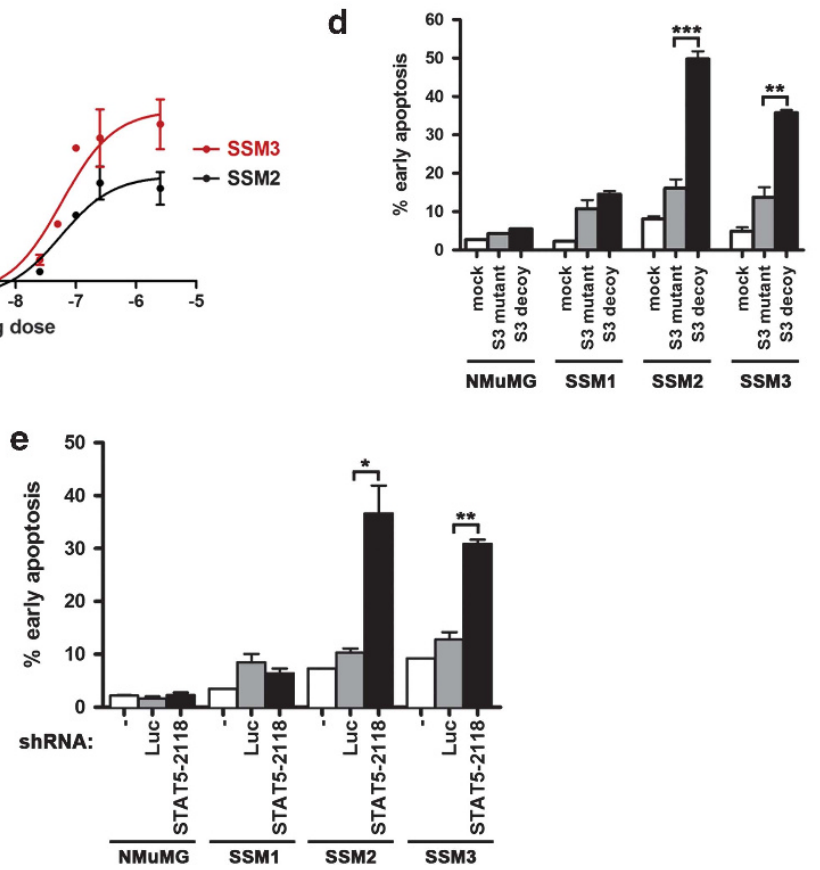

Figure 3 Persistently active PrIR-JAK2-STAT3/5A/5B signaling confers a survival signal. (a) Reduction in PrIR expression by PrlR-specific shRNA hairpins led to a significant increase in the percentage of early apoptotic cells in SSM2 and SSM3, as measured by flow cytometry. Luc, luciferase-specific shRNA. (b) JAK2-specific shRNA hairpins induced apoptosis in SSM2 and SSM3 compared with control Luc shRNA, as measured by flow cytometry. (c) BMS-911543 induced a dose-dependent increase in apoptosis in SSM2 and SSM3. IC ${ }_{50}=56 \mathrm{nM}$ for SSM2 and $58 \mathrm{nM}$ for SSM3. (d) Blockade of STAT3 function using a double-stranded oligonucleotide (S3 decoy) induced apoptosis in SSM2 and SSM3. A mutant oligo (S3 mutant) had no significant effect on cell survival. (e) STAT5A/5B knockdown resulted in an increase in early apoptosis over control hairpin-transduced SSM2 or SSM3 cells. ${ }^{*} P<0.05,{ }^{\star \star} P<0.01,{ }^{* \star *} P<0.001$. Error bars, S.E.M.

the frequency of luminal progenitors was $84 \%$ in STAT1 ${ }^{-1-}$ mammary glands compared with $59 \%$ in WT glands (Figure 5d). STAT1 ${ }^{-1}$ - luminal epithelial cells also produced 1.5-fold more mammosphere colonies in culture than their WT counterparts (Figure 5e). Thus, STAT1 ${ }^{-/}$- mammary glands contain a larger proportion of luminal progenitors that are presumably better equipped for self-renewal than WT epithelium. The percentage of CD49f ${ }^{\text {int }} \mathrm{CD} 24^{\text {hi }} \mathrm{CD} 61^{+}$cells dropped from 84 to $64 \%$ in mammary glands of BMS-911543treated STAT1 ${ }^{-1-}$ mice and the number of mammosphere colonies also showed a $77 \%$ decrease (Figures $5 \mathrm{~d}$ and e). Thus, activated JAK2 is responsible for the aberrant increase in the proportion of luminal progenitors and the enhanced selfrenewal capacity in STAT1 ${ }^{-1}-$ mammary glands.

To examine whether luminal progenitors represent the precursor cells that initiate tumor development, we analyzed the surface expression level of CD61 on STAT1-1mammary tumor cells. CD49f ${ }^{\text {int }}$ CD2 $44^{\text {hi }}$ tumor cells expressed high levels of CD61 (Figure 5f). Interestingly, these CD61 ${ }^{+}$ tumor cells were also positive for PrIR. Results from transplantation studies indicated that $\mathrm{CD} 61^{+} \mathrm{PrIR}^{+}$tumor cells were enriched for tumor-initiating cells (Supplementary Table S2). These results strongly suggest that STAT1 ${ }^{-1-}$ $\mathrm{ER} \alpha^{+}$mammary tumors originate from luminal progenitors.

To confirm that JAK2-dependent expansion of luminal progenitors causes tumorigenesis in STAT1 ${ }^{-1}$ mammary glands, we treated STAT1 ${ }^{-1}$ - mice with BMS-911543 before clinically apparent disease was detected. Remarkably, none of the 18 mice treated with BMS-911543 developed mammary gland adenocarcinomas (Figure $5 \mathrm{~g}$ ). In stark contrast, 8/12 $(67 \%)$ of vehicle-treated STAT1 ${ }^{-1-}$ mice developed tumors (consistent with our previous findings ${ }^{18}$ ). No toxicity was 
a

\section{悉}



vehicle



BMS-911543

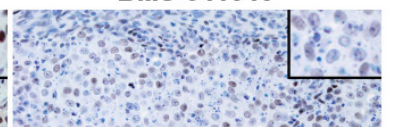

b

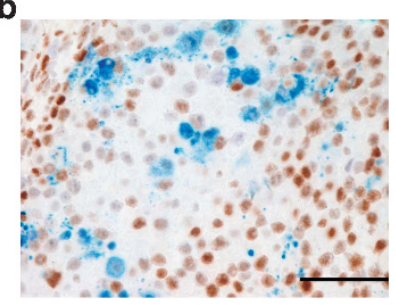

c

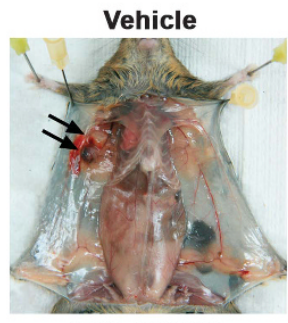

BMS-911543

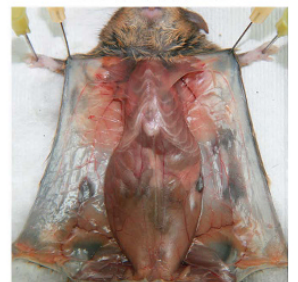

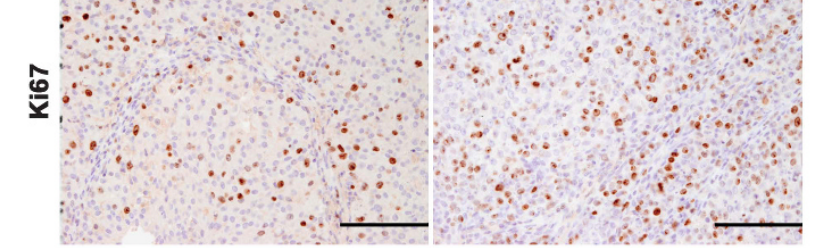

d





Figure 4 Systemic JAK2 inhibition abrogated growth of primary and ovarian hormone-independent STAT1 ${ }^{-1-}$ mammary tumors. (a-d) STAT1 ${ }^{-1-}$ mice bearing primary mammary tumors at $5 \mathrm{~mm}$ in diameter were administered vehicle or BMS-911543 at $10 \mathrm{mg} / \mathrm{kg}$ once-daily by oral gavage. (a) pSTAT3, pSTAT5A/5B, cleaved caspase 3 and Ki67 levels were examined by immunohistochemical analyses on tumors harvested from vehicle or BMS-911543-treated mice at $6 \mathrm{~h} \mathrm{post-gavage.} \mathrm{Scale} \mathrm{bar}=100 \mu \mathrm{m}$. Original magnification $=\times 200$. Inset $=\times 600$. (b) Double immunohistochemical analysis of pSTAT3 (brown) and cleaved caspase 3 (blue) on a tumor section from a STAT1 $^{-1-}$ mouse treated with a single dose of BMS-911543 and killed $3 \mathrm{~h}$ post-gavage. Positive caspase 3 signal (blue) was enriched in areas lacking pSTAT3 (brown). Scale bar $=50 \mu \mathrm{m}$. (c) Representative vehicle and BMS-911543-treated mice after 26 days of treatment. Arrows indicate primary tumors in the vehicle-treated mouse. (d) Growth curves of primary STAT1 ${ }^{-1}$ mammary tumors in vehicle- or BMS-911543-treated mice. To underscore the changes in tumor size at the start of the study, mean tumor diameter is shown. vehicle, $n=7$; BMS-911543, $n=16$. (e) BMS-911543 abrogated the progression of ovarian hormone-independent tumors. vehicle, $n=3$; BMS-911543, $n=4$. Data from one ovarian hormone-independent tumor line derived from Figure 1a is shown. See also Supplementary Figure S3 for two other tumor lines. ${ }^{* * *} P<0.0001$. Error bars, S.E.M.

observed in the BMS-911543-treated cohort (Supplementary Figures S4B-S4D). Together these findings demonstrate that (a) inhibition of JAK2 signaling through the prophylactic use of a pharmacological inhibitor prevents tumor development and (b) persistent activation of JAK2 increases the luminal progenitor population and drives mammary tumor formation in the absence of STAT1.

Activation of JAK2 and STAT3 in human breast cancers. To generalize our findings to humans, we examined JAK2 and STAT3 activation in ER $\alpha^{+}$breast cancer biopsies by immunohistochemistry. pJAK2 and pSTAT3 were observed in human breast cancer cells in patterns similar to those in STAT1 ${ }^{-1-}$ mammary tumors (Figure 6). In addition, JAK2 and STAT3 activation occurred early during tumor progression as in situ lesions were positive for both pJAK2 and pSTAT3. In cases where co-activation of JAK2 and STAT3 was found, STAT1 expression was greatly suppressed in the neoplastic human cells, resulting in a functional condition that recapitulates the phenotypes seen in STAT1 ${ }^{-1-} \mathrm{ER} \alpha^{+}$mammary tumors. 


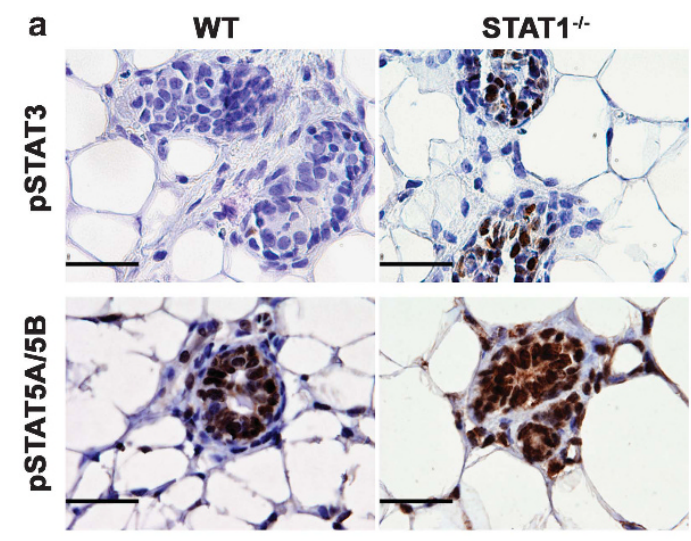

b


STAT1 $\%$
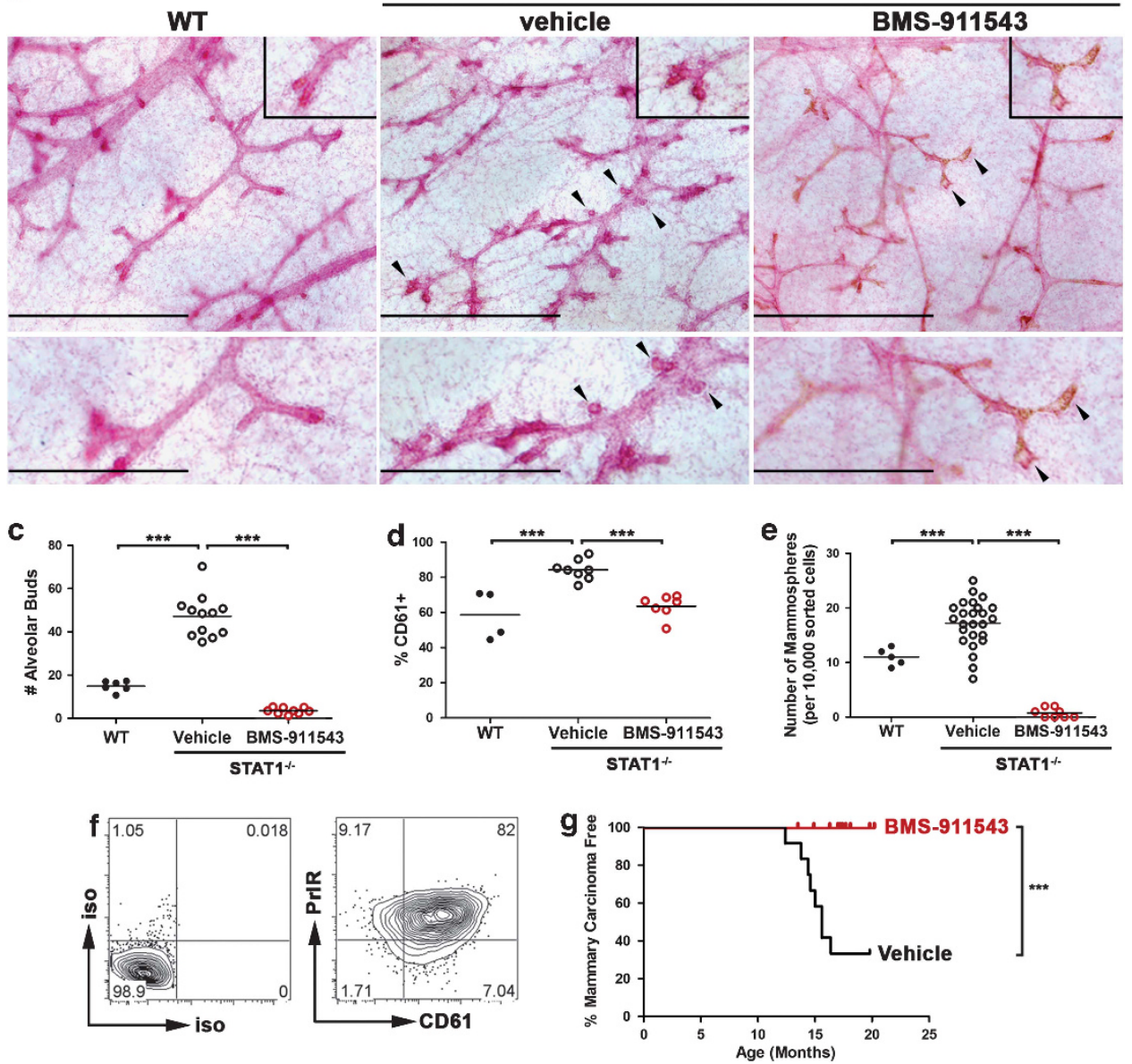

Figure 5 JAK2 activation causes an expansion of luminal progenitors and drives mammary tumor development in STAT1 ${ }^{-1-}$ mice. (a) Immunohistochemical analysis of pSTAT3 and pSTAT5A/5B in mammary glands from age- and estrus-matched WT and STAT1 ${ }^{-1}$ - mice. Scale bar $=33 \mu \mathrm{m}$. (b) Whole-mount analysis of mammary glands from age- and estrus-matched WT and STAT1 ${ }^{-1-}$ mice. BMS-911543 treatment significantly reduced the number of alveolar buds (arrowheads). Representative images of four to nine mice per group. Original magnification $=\times 40$. Inset $=\times 80$. Scale bar $=1 \mathrm{~mm}$ (top), $0.5 \mathrm{~mm}$ (bottom). (c) Quantification of the number of alveolar buds per high-power field $(\times 100)$ of whole-mount mammary glands. (d) The percentages of $\mathrm{CD} 61^{+}$progenitor cells in the CD49f ${ }^{\text {low }} \mathrm{CD} 24^{\text {hi }}$ luminal epithelial subset were quantified in WT, and vehicle- and BMS-911543-treated STAT1 ${ }^{-1}$ mammary glands. (e) CD49flow CD24 ${ }^{\text {hi }}$ luminal epithelial cells from WT and STAT1 ${ }^{-1-}$ mice were sorted and cultured in mammosphere media (a measure of self-renewal capacity). (c-e) ${ }^{* \star *} P<0.0001$. (f) CD4gf ${ }^{\text {int }}$ CD24 ${ }^{\text {hi }}$ STAT1 ${ }^{-1-}$ mammary tumor cells co-expressed PrIR (PrIR MAb 5A12) and the luminal progenitor marker CD61. Isotype controls (Iso) were used to define positive gates. Representative plots from three independent tumors. (g) Long-term administration of BMS-911543 to STAT1 ${ }^{-i}$ mice before the development of clinically apparent disease prevented mammary tumor formation. Vehicle, $n=12$; BMS-911543, $n=18 .{ }^{* *} P<0.001$

STAT1-induced SOCS1 antagonizes JAK2-STAT3/5A/5B signaling. The observations that dysregulated JAK2STAT3/5A/5B signaling was detected in STAT1-deficient
MECs prompted us to explore the possibility that STAT1 antagonized this pathway. We previously showed that STAT1's tumor-suppressor function was mediated by its 


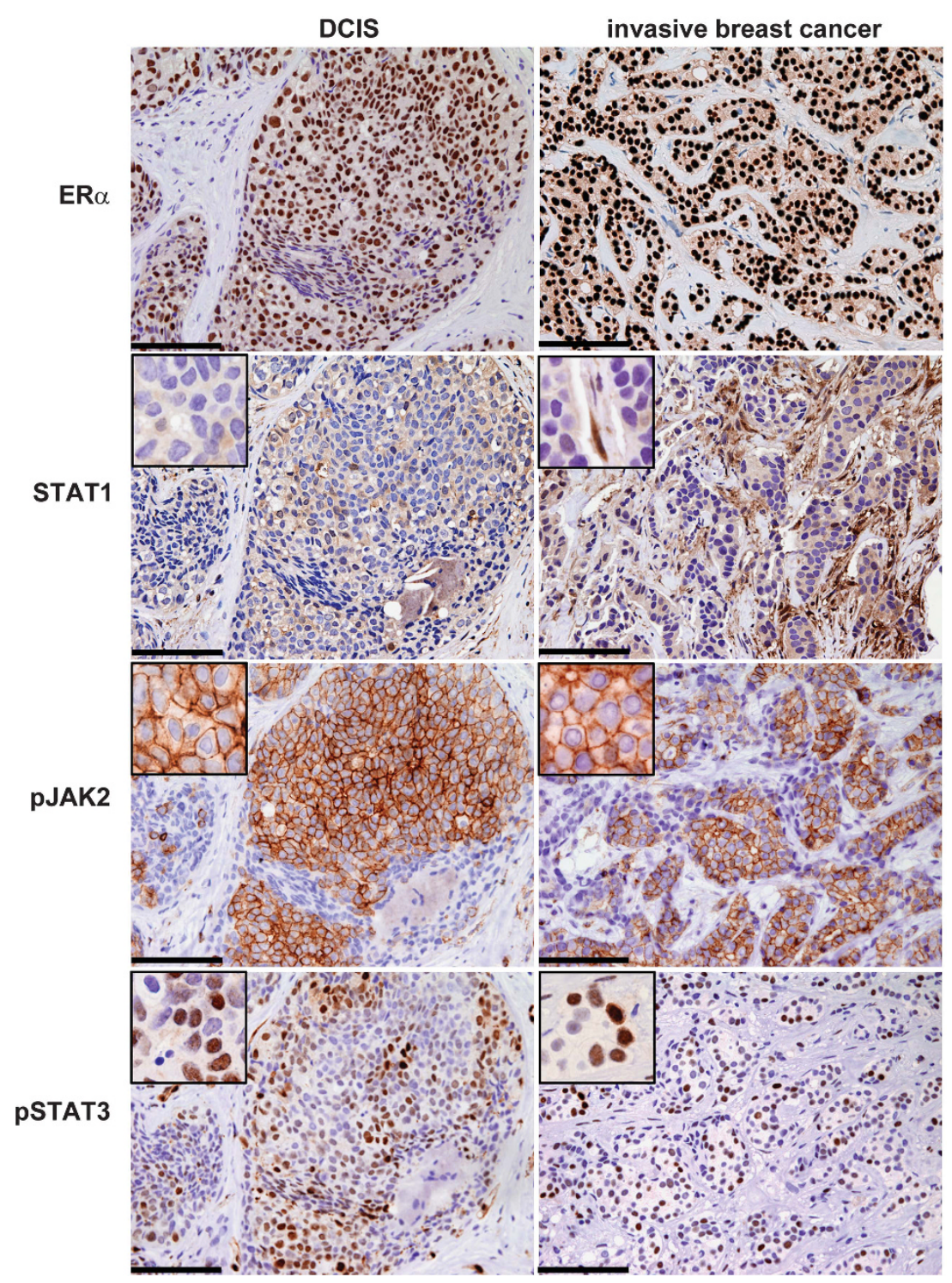

Figure 6 Co-activation of JAK2 and STAT3 in human ER $\alpha^{+}$breast cancer cells that have diminished STAT1 expression. Representative images of ER $\alpha^{+}$ductal carcinoma in situ (DCIS) and ER $\alpha^{+}$invasive breast cancer showing a lack of STAT1 expression in the neoplastic cells but the presence of phosphorylated JAK2 (pJAK2) and STAT3 (pSTAT3). Insets highlight the cellular localization of pJAK2 (cell plasma membrane) and pSTAT3 (nuclei). Original magnification, $\times 200$. Inset, $\times 600$. Scale bar $=100 \mu \mathrm{m}$

transcription factor activity because only WT STAT1, but not a transcriptionally inactive Y701F STAT1 mutant, reverted the tumorigenic phenotype of STAT1 ${ }^{-1}$ mammary tumor cells and induced tumor cell apoptosis. ${ }^{18}$ We now show that STAT1 undergoes Y701 phosphorylation before apoptosis (Figure 7a and Supplementary Figure S5). STAT1 phosphorylation was abrogated in STAT1-transduced SSM2 or SSM3 cells treated with BMS-911543 (Figure 7b). Therefore, JAK2 activation is required to phosphorylate ectopically expressed STAT1 in STAT1 ${ }^{-/}$mammary tumor cells. Activation of transduced WT STAT1 in SSM2 and SSM3 cells led to upregulation of the STAT1-regulated target genes, IRF1 and SOCS1 (Figures 7c and d). In contrast, expression of the Y701F STAT1 mutant did not. Together, these observations demonstrate that activation of STAT1 by JAK2 and the induction of target gene expression are important steps in conferring the tumor-suppressor function of STAT1.

Under normal physiological conditions, the growth signal emanating from PrIR is terminated by the regulatory action of the SOCS proteins. ${ }^{29-34}$ We tested whether the inability of STAT1 ${ }^{-1-}$ mammary tumor cells to restrain activation of PrIR-JAK2-STAT3/5A/5B was due to a lack of negative regulation by the SOCS proteins and whether introduction of STAT1 could restore that checkpoint. SOCS2 and SOCS3 are highly expressed in STAT1 ${ }^{-1-}$ mammary tumor cells (Figure 1) and thus are insufficient to regulate PrIR signaling in these cells. In contrast, SOCS1 is absent in STAT1 ${ }^{-/-}$ mammary tumor cells but is robustly upregulated following ectopic STAT1 expression (Figure 7d). Importantly, introduction of SOCS1 into SSM2 and SSM3 markedly reduced the levels of pSTAT3 and pSTAT5A/5B (Figure 7e) and 



g
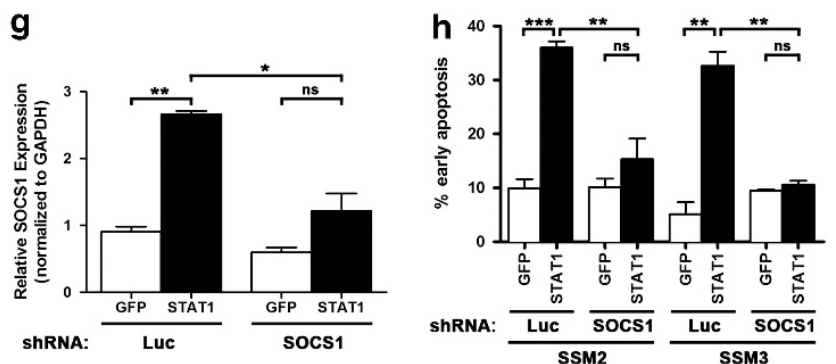

Figure 7 STAT1 activation antagonizes PrIR-JAK2-STAT3/5A/5B activation by inducing SOCS1 expression. (a) pSTAT1 levels were quantified in NMuMG, SSM1, SSM2 and SSM3 following STAT1 transduction. Geometric mean channel shift (GMCS) was calculated by subtracting the GM obtained with the isotype control from that with the anti-pSTAT1 Ab. (b) JAK2 inhibition by BMS-911543 abrogated STAT1 phosphorylation in SSM2 and SSM3 following STAT1 enforced expression. (c and d) Expression of STAT1 in SSM2 and SSM3 resulted in the upregulation of STAT1-regulated genes, IRF1 (c) and SOCS1 (d). STAT1 mutant Y701F failed to upregulate IRF1 and SOCS1. (e) Retrovirus expressing GFP or SOCS1.GFP was transduced into SSM2 or SSM3. SOCS1 inhibited PSTAT3 and pSTAT5A/B in SSM2 and SSM3, as measured by flow cytometry. A rabbit (Rb) lgG was used as an isotype control (leftmost). Contour plots are representative of three independent experiments. (f) Enforced expression of SOCS1 in SSM2 and SSM3 induced apoptosis, but had no effect on NMuMG and SSM1. (g) SSM2 cells were transduced with a Luc or SOCS1-specific shRNA. SSM2 cells were then transduced with GFP or STAT1. SOCS1 induction was measured by RT-PCR. (h) In SSM2 and SSM3 cells stably expressing a SOCS1-specific shRNA hairpin (SOCS1), ectopic expression of STAT1 failed to cause tumor cell death, in contrast to SSM2 and SSM3 cells expressing control Luc shRNA. ${ }^{\star} P<0.05,{ }^{\star \star} P<0.01,{ }^{* \star} P<0.001$. Error bars, S.E.M.

subsequently induced tumor cell apoptosis (Figure 7f). Therefore, SOCS1 appears to have a critical role in regulating STAT3/ $5 \mathrm{~A} / 5 \mathrm{~B}$ activation and terminating the survival signal.

To directly test whether STAT1-induced apoptosis requires SOCS1, we examined whether concomitant knockdown of SOCS1 could block STAT1-mediated apoptosis (Figure 7g). STAT1 expression in control shRNA-expressing SSM2 and
SSM3 significantly increased the percentage of apoptotic cells (Figure 7h). In contrast, ectopically expressed STAT1 failed to induce tumor cell death in cells stably expressing SOCS1 shRNA. Together, these data reveal that STAT1 suppresses mammary tumor formation by transcriptionally inducing SOCS1, which in turn has a critical role in regulating PrIRJAK2-STAT3/5A/5B signaling. 


\section{Discussion}

Although the role of PrIR signaling in mammary gland development has been extensively studied, its action in development of $\mathrm{ER} \alpha^{+}$breast cancer remains elusive. The observations that (a) human $\mathrm{ER} \alpha^{+}$breast cancer cells frequently suppress STAT1 expression but upregulate PrIR and (b) STAT1 ${ }^{-1-}$ mice spontaneously develop ER $\alpha^{+} \mathrm{PR}^{+}$ $\mathrm{PrIR}^{+}$mammary tumors provided us with a unique opportunity to investigate the causality of PrIR signaling in $\mathrm{ER} \alpha^{+}$ tumor development. ${ }^{4,5,18}$ Our data show that dysregulated PrIR signaling initiates and drives outgrowth of $\mathrm{ER} \alpha^{+}$ mammary tumors. Specifically, dysregulated PrIR signaling occurs in nontransformed MECs of STAT1 ${ }^{-/}$mice and promotes survival and expansion of $\mathrm{CD} 61^{+}$luminal progenitors that eventually develop into $\mathrm{ER} \alpha^{+}$mammary tumors. We show that STAT1 fulfills a tumor-suppressor function by inducing SOCS1, which in turn inhibits JAK2 (ref. 35) and thereby prevents the normal developmental functions of STAT3 and STAT5A/5B from becoming pro-oncogenic (Figure 8). In nascent tumor cells that have downregulated STAT1 or SOCS1 expression, ${ }^{18,36}$ activation of JAK2-STAT3/5A/5B is left unchecked. The lack of this physiologically important cellular checkpoint results in mammary tumor formation.

Our results reveal that STAT1 is critical in regulating the pro-survival effects of PrIR signaling in both homeostatic and pathological settings. Overabundance of alveolar buds, $\mathrm{CD} 61^{+}$luminal progenitors, and the self-renewal capability of luminal epithelial cells are observed in STAT1 ${ }^{-1-}$ mammary glands before transformation. These effects are not a consequence of differential systemic hormone production or local production of Prl. Our observations are therefore consistent with the conclusion that the abnormality in $\mathrm{STAT1}^{-/-}$mammary glands lies in the inability to control PrIR signaling at the level of the epithelial cells. These results are also supported by previous findings that maintenance of $\mathrm{CD} 61^{+}$luminal progenitors requires mammary gland-specific expression of STAT5A. ${ }^{37}$ In addition, we show that JAK2 inhibition reverses the phenotypes in STAT1 ${ }^{-/}$mammary glands and prevents the emergence of tumor cells that co-express CD61 and PrIR. Transplantation studies confirm $\mathrm{CD} 1^{+} \mathrm{PrIR}^{+}$tumor cells as the tumor-initiating cells.
Therefore, we propose that luminal progenitors represent the precursor cells of STAT1 ${ }^{-1-} \mathrm{ER} \alpha^{+}$mammary tumors.

Importantly, our STAT1 ${ }^{-1-}$ model of ER $\alpha^{+}$breast cancer is mechanistically distinct from other published $\mathrm{ER} \alpha^{+}$models. Specifically, it does not depend on over-production of $\mathrm{Prl}$ ligand and thus differs from the NRL-PRL model where augmented transgenic $\mathrm{Prl}$ production is critical to tumor development. ${ }^{38}$ Although JAK2 is required for initiation of the NRL-PRL tumors, it is not essential for tumor maintenance. $^{39}$ In contrast, STAT1 ${ }^{-1-}$ tumors require JAK2 activation for both initiation and progression. The $\mathrm{STAT}^{-1-}$ model is also distinct from the transgenic ER $\alpha$ CERM model where dysregulated ER $\alpha$ expression promotes hyperproliferation of MECs and development of ductal carcinoma in situ, but is not sufficient to drive invasive carcinoma development. ${ }^{40}$ Our findings that persistent PrIR-JAK2-STAT3/5A/5B signaling confers a survival signal suggest that dysregulated JAK2 signaling may collaborate with $\mathrm{ER} \alpha$ signaling to drive the entire tumorigenesis process. Consistent with this notion, the constitutively active JAK2 mutant (V617F) accelerates tumorigenesis with the collaboration of other oncogenes. ${ }^{41}$

We further show that the molecular mechanism whereby STAT1 exerts its tumor-suppressor function is through the transcriptional regulation of SOCS1. Specifically, concomitant enforced expression of STAT1 with blockade of SOCS1 induction prevents STAT1-mediated cell death. Therefore, the STAT1-SOCS1 axis counteracts the pro-survival effect of STAT3/5A/5B. Our findings are consistent with the currently held paradigm of STAT1 activation in the developmental cycle of murine mammary glands. STAT1 activation reaches highest levels in mature, fully developed quiescent mammary glands when STAT5 is activated at a basal level, ${ }^{42}$ and again late during mammary gland involution when tissue homeostasis returns to a relatively quiescent state. $^{43,44}$ Our observations therefore provide important insights into the role of STAT1-induced SOCS1 in controlling PrIR-mediated tumor cell development. As IRF1 induction by STAT1 is critical for SOCS1 expression, ${ }^{45,46}$ it follows that STAT1's tumor-suppressor function would be IRF1 dependent. $^{19}$

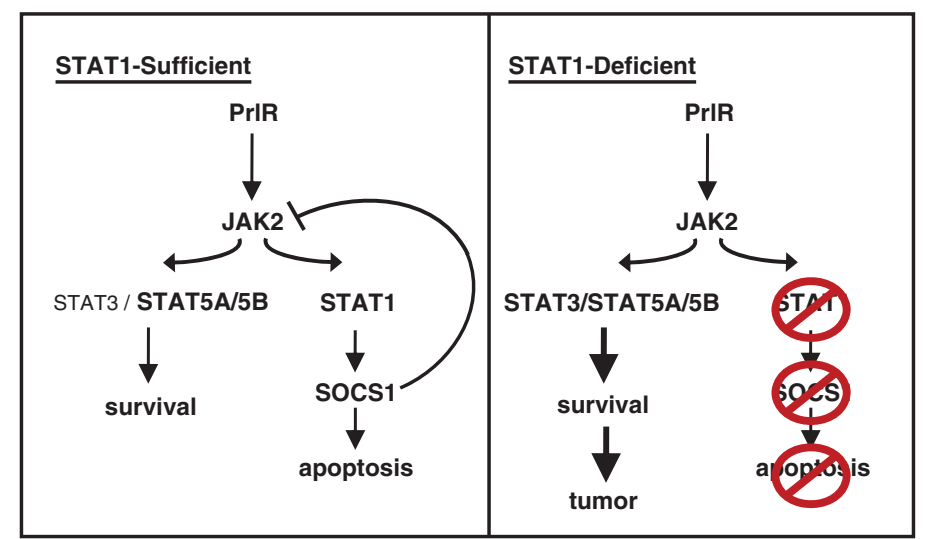

Figure 8 Graphical summary of the molecular mechanism of the tumor-suppressor function of STAT1-SOCS1. In the presence of STAT1, JAK2 activation is normally regulated by SOCS1, which is transcriptionally induced by STAT1. In MECs that have downregulated STAT1 or are unable to upregulate SOCS1, persistent JAK2 activation results in unopposed STAT3/5A/5B activation, promotes survival of MECs, and drives development of ER $\alpha^{+}$mammary tumors. See Discussion section for details 
It is noteworthy that both STAT3 and STAT5A/5B are phosphorylated by PrIR-JAK2 activation in STAT1 ${ }^{-1}$ - tumor cells despite a dominant activation of STAT5A/5B in normal WT MECs. The loss of STAT1-SOCS1 regulation on PrIR may promote a more significant activation of STAT3 ultimately altering the ratio of activated STAT3 and STAT5A/5B. More detailed gene expression profiling analyses are needed to distinguish the precise targets of these STATs in ER $\alpha^{+}$ tumors. Our functional data underscore the importance of both STAT3 and STAT5A/5B in promoting tumor cell survival. Therefore, in this neoplastic setting with dysregulated JAK2 activity, STAT3 and STAT5A/5B appear to mediate similar biological functions. Consequently, JAK2 blockade is more robust than inhibiting STAT3 or STAT5 alone.

Our results also show that PrIR-JAK2-STAT3/5A/5B activation is highly prevalent in human $\mathrm{ER} \alpha^{+}$breast cancers and thus a potential druggable pathway. Several approaches that include suppressing Prl production, inhibiting ligandreceptor binding and disrupting the transcription factors necessary for signaling have not been translated into successful therapies. ${ }^{47}$ Our findings demonstrate, for the first time, that ablating PrIR signaling by JAK2-targeted inhibition exhibits efficacy in treating primary and endocrine refractory $\mathrm{ER} \alpha^{+}$mammary tumors. Our results also indicate that PrIRJAK2 activation is a stronger driver of tumor progression than ligand-dependent $\mathrm{ER} \alpha$ signaling as JAK2 inhibition prevents the outgrowth of resistant tumors while ovarian ablation does not. Therefore, JAK2 inhibition may offer a novel, effective alternative to or combination with traditional endocrine treatments for $\mathrm{ER} \alpha^{+}$breast cancer. It is also noteworthy that STAT3 activation is observed in stem cell-like cancer cells from different molecular subtypes of breast cancer and targeting JAK2 is effective to treat the triple-negative subtype in preclinical models. ${ }^{48-50}$ Together, these results suggest a pivotal role for JAK2 irrespective of the receptors mediating activation and thus underscore a generalizable potential for JAK2-targeted therapy to treat multiple forms of this disease.

In summary, our findings elucidate the cellular and molecular mechanisms whereby STAT1 suppresses ER $\alpha^{+}$ tumor development in the mammary gland. The data presented herein thus provide a platform to develop novel therapies that may be effective in treating human $\mathrm{ER} \alpha^{+}$ breast cancers.

\footnotetext{
Materials and Methods

Mice. STAT $1^{-l-}$ mice have been previously characterized in our laboratory. ${ }^{18}$ WT 129S6/SvEv and STAT1 ${ }^{-1-}$ mice were purchased from Taconic Farms (Hudson, NY, USA). Characterization of the small molecule JAK2 inhibitor BMS911543 was previously described. ${ }^{22}$ To determine whether JAK2 activation was required to maintain tumor growth, STAT1 ${ }^{-1-}$ mice bearing primary mammary tumors around $5 \mathrm{~mm}$ in diameter were treated with BMS-911543 at $10 \mathrm{mg} / \mathrm{kg}$ in PEG490/citrate buffer $(80: 20)$ once daily by oral gavage. Tumors were measured at two perpendicular diameters every 3-4 days and the average diameters were used for analyses. Pharmacokinetic analysis was performed in tumor-bearing mice post-treatment (Supplementary Table S1). Concentration of the inhibitor in mouse serum was consistent with levels shown to fully suppress the constitutively active JAK2 pathway in vivo. ${ }^{22}$ To determine whether JAK2 activation was required to drive tumor development in STAT1 ${ }^{-1}$ mice, tumor-free STAT1 ${ }^{-1-}$ mice approximately 8 months of age were administered BMS-911543 at $10 \mathrm{mg} / \mathrm{kg}$ or vehicle PEG490/citrate buffer $(80: 20)$ daily by oral gavage. Mice were scored as tumor bearing when masses reached $5 \times 5 \mathrm{~mm}^{2}$ in size and continued to progress. All animal experiments were carried out according to the guidelines of the American
}

Association for Laboratory Animal Science under an approved protocol by the Animal Studies Committees and performed in AAALAC-accredited specific pathogen-free facilities at Washington University School of Medicine in St. Louis, MO, USA.

Cell cultures. The maintenance of the SSM1, SSM2 and SSM3, and NMuMG cell lines has been described elsewhere. ${ }^{18}$

Quantitative real-time PCR. One microgram of DNase I-treated total RNA was used to synthesize first strand CDNA using SuperScript RT III and random primers (Invitrogen, Grand Island, NY, USA). qRT-PCR was carried out using gene-specific Taqman primers and probes, and an ABI Prism 7000 (Applied Biosystems, Grand Island, NY, USA).

Immunohistochemistry on mouse tissues. Four-micron sections of formalin-fixed paraffin-embedded mammary glands from WT and STAT1 ${ }^{-1-}$ mice or STAT1 ${ }^{-1-}$ mammary tumors were stained using antibodies against the phosphorylated form of JAK2 (pJAK2; rabbit monoclonal E132, Abcam, Cambridge, MA, USA), STAT3 (pSTAT3; rabbit monoclonal D3A7, Cell Signaling, Boston, MA, USA) and STAT5 (pSTAT5; rabbit monoclonal E208, Abcam), cleaved caspase 3 (rabbit polyclonal, R\&D Systems, Minneapolis, MN, USA), Ki67 (clone TEC-3, Dako, Carpinteria, CA, USA) and phospho-histone $\mathrm{H} 3$ (rabbit polyclonal S10, Abcam). As specificity controls, sections were stained in the absence of primary antibody or preincubated with a blocking phospho-peptide before the addition of the anti-pJAK2 $M A b$. Upon antigen retrieval and incubation of the primary antibody, positive signal was revealed by EnVision System HRP (Dako) followed by DAB chromogen. For double stain, Mach4 AP-polymer detection system followed by Ferangi Blue chromogen (Biocare Medical, Concord, CA, USA) was used. Images were captured using an Olympus DP-70 digital camera mounted on an Olympus BX60 microscope (Center Valley, PA, USA).

Immunohistochemistry on human breast cancer biopsies. Formalin-fixed paraffin-embedded diagnostic core biopsies were from the archive of the Department of Pathology at the University of Brescia, Brescia, Italy. This retrospective study was conducted in compliance with the Helsinki Declaration and with policies approved by the Ethics Board of Spedali Civili di Brescia. The cohort consisted of diagnostic core biopsies from $40 \mathrm{ER}^{+} \mathrm{PR}^{+}$invasive breast carcinomas and 5 ER + PR + in situ lesions collected between 2008 and 2012. To preserve post-translational modification of phosphoproteins in human tissues, breast core biopsies were immersed in buffered formalin fixative immediately upon removal from patients. Immunohistochemical analysis of pJAK2 and pSTAT3 was performed as described in the previous section. STAT1 expression was assessed according to procedures reported previously. ${ }^{18}$

Electrophoretic mobility shift assay. EMSA was performed as previously described. ${ }^{51}$ Oligos used are as follows: (m67 sense) $5^{\prime}$-GAGTGA CATTTCCCGTAAATCAT-3', (m67 antisense) 5'-GAGTATGATTTACGG GAAATGTC-3', ${ }^{2}$ (STAT5 sense) 5'-GAGTCGAATTCCAGGAATTCA-3', (STAT5 antisense) $5^{\prime}$-GAGTTGAATTCCTGGAATTCG-3'. For supershift assays, $1 \mu \mathrm{g}$ of STAT3 (Zymed, Grand Island, NY, USA; 13-7000) or STAT5 (Santa Cruz Biotechnology, Dallas, TX, USA; sc-835) antibodies was incubated with the nuclear extracts for $1 \mathrm{~h}$ at $4{ }^{\circ} \mathrm{C}$ before the addition of the DNA probes.

Flow cytometry analysis of $\mathbf{H}-2 \mathbf{K}^{\mathbf{b}}$ MHC Class I. SSM2 tumor cells were transduced with the two PrIR-specific shRNA hairpins. Surface expression of $\mathrm{H}-2 \mathrm{~K}^{\mathrm{b}} \mathrm{MHC}$ class I was quantified using anti-H-2K $\mathrm{K}^{\mathrm{b}}-\mathrm{PE}$ (BD Biosciences, San Jose, CA, USA).

Immunoprecipitation and western blotting. SSM1, SSM2, SSM3 and NMUMG cells were lysed in RIPA buffer with $2 \mathrm{mM}$ sodium vanadate, protease inhibitor cocktail (Sigma, St Louis, MO, USA) and phosphatase inhibitor cocktail (Sigma). One milligram of lysate was pre-cleared with protein A (GE Healthcare, Waukesha, WI, USA) and incubated with $1 \mu \mathrm{g}$ of antibodies (all from Santa Cruz Biotechnology; STAT3, sc-483; STAT5, sc-835; JAK1, sc-277; JAK2, sc-278) for $16 \mathrm{~h}$ at $4{ }^{\circ} \mathrm{C}$. Immunoprecipitates were pulled down by protein $\mathrm{A}$, washed extensively and resolved on SDS-PAGE. Phospho-tyrosine was detected by antibody against pTyr (Millipore, clone 4G10, Billerica, MA, USA). Antibody specific for the phospho-tyrosine 1007/1008 of JAK2 was used for the detection of kinase active JAK2 (Cell Signaling). 
shRNA-mediated knockdown. All shRNA hairpins were cloned into pFLRU lentivirus vector (generous gift from Dr. Greg Longmore, Washington University in St. Louis). The sequences of the two PrIR shRNA hairpins are as follows: PrIR-292, 5'-GTAAATGCCACGAACGCCA-3'; PrIR-1374, 5'-GGAA GAAGTGGCTGTGCAAGA-3'. Two independent JAK2 shRNA hairpins were used: JAK2-99, $5^{\prime}$-GCAGATAGAGCCAGTCCTTCA-3' (this study), and JAK2-225, $5^{\prime}$-GGAGAGTATCTGAAGTTTC- $3^{\prime}{ }^{53}$ The sequence of the shRNA hairpin that targets both STAT5A and STAT5B is $5^{\prime}$-GGGTTTCGTGAACAAGCAACA- $3^{\prime}$. The SOCS1 shRNA hairpin, 5'-GCCGCTGTGCCGCAGCATTAA-3', was cloned into a pFLRU plasmid carrying the mCherry coding sequence. SOCS1 shRNAexpressing cells were sorted using the mCherry marker. Lentivirus was prepared by transfecting $293 \mathrm{~T}$ cells with the pFLRU plasmid, $\Delta 8.2$ and pCMV.VSVg. The infected cells were harvested on day 2 post-infection for qRT-PCR and western blotting to determine knockdown efficiency, on day 2.5 for flow cytometric analysis of phosphorylated STAT3 and STAT5, and on day 3 for apoptosis assay.

STAT1 and SOCS1 expression. Retrovirus expressing GFP alone, STAT1.IRES.GFP, STAT1 mutant Y701F.IRES.GFP, SOCS1.IRES.GFP was prepared by co-transfecting Phoenix cells with the retrovirus plasmid and pCMV.VSVg using FUGENE HD (Promega, Madison, WI, USA). Supernatant was harvested 48 and $72 \mathrm{~h}$ post-transfection, and overlayed on NMuMG, SSM1, SSM2 or SSM3 cells in the presence of $8 \mu \mathrm{g} / \mathrm{ml}$ of polybrene for 6 to $8 \mathrm{~h}$. Infection was carried out for 2 consecutive days. The infected cells were harvested on day 2 post-infection for western blotting to confirm expression, day 2.5 for qRT-PCR for IRF1 and SOCS1, and on day 3 for flow cytometry to quantitate the percentages of cells undergoing early apoptosis, or exhibiting tyrosine phosphorylated STAT1, STAT3 or STAT5. To determine whether JAK2 phosphorylated STAT1, STAT1 reconstituted SSM2 and SSM3 cells were treated with $125 \mathrm{nM}$ of the JAK2 inhibitor BMS-911543 (ref. 22) for $14 \mathrm{~h}$. DMSO- and BMS-911543-treated cells were analyzed by flow cytometry for STAT1 tyrosine phosphorylation.

Blockade of STAT3 function by double-stranded (ds) oligonucleotide decoy. The STAT3-binding site decoy is a short ds oligonucleotide that binds selectively to the DNA-binding domain of activated STAT3 homodimers, thereby blocking the induction of STAT3 target genes. ${ }^{25}$ A mutant oligonucleotide that differs from the STAT3 decoy by a single nucleotide is used as control.

Mammosphere assay. Single-cell suspension was prepared from the inguinal mammary fat pads of 8-month-old WT, and vehicle- or BMS-911543-treated $\mathrm{STAT}^{-1}{ }^{-}$- female mice as described above. CD49f ${ }^{\text {int }} \mathrm{CD} 24^{\text {hi }}$ luminal epithelial cells depleted of DAPI, CD31, CD45 and TER119 were sorted using an Aria cell sorter (BD Biosciences) and plated at the concentration of 10000 cells per well in 24-well ultralow attachment plates (Corning, Corning, NY, USA) in mammosphere media (1:1 DMEM:F12/1X B27 (Invitrogen)/20 ng/ml mEGF (BD Biosciences) $/ 20 \mathrm{ng} / \mathrm{ml}$ bFGF (BD Biosciences) $/ 1 \%$ penicillin-streptomycin. ${ }^{54}$ The purity of the sorted cells was higher than $85 \%$. Only colonies larger than $50 \mu \mathrm{m}$ were counted 8 days after plating.

Transplantation into fat pad. After cell sorting, the designated number of cells were washed once with phenol red-free DMEM, resuspended in phenol redfree DMEM and transplanted into the inguinal fat pads of female STAT1 ${ }^{-1-}$ mice. Mice were monitored until palpable tumors developed or up to 12 months if no tumors were observed.

Flow cytometry. Quantification of PrIR expression was done using biotinylated $\mathrm{Prl}(\mathrm{b}-\mathrm{Prl})$. Briefly, recombinant murine Prl (purchased from the National Hormone and Peptide Program) was biotinylated using Enzotin with the Enzotin to Prl ratio 12: 100 following the manufacturer's specifications (Enzo Biochem, Farmingdale, NY, USA). Binding of biotinylated Prl to cell surfaces was specific (i.e., it could be completed by addition of non-biotinylated Prl ligand (Supplementary Figure S1A)) and saturable (data not shown). For the Prl competition assay, varying amounts of nonbiotinylated Prl were added to SSM2 tumor cells $(0.01,0.1,1,5$ and $10 \mu \mathrm{g})$ before the addition of b-Prl. For flow cytometry analysis of primary STAT1 ${ }^{-1}$ mammary tumors, tumors were disaggregated as described previously. ${ }^{18}$ Cells were first blocked with normal rat serum, anti-CD16 and anti-CD32 Fc $\gamma$ receptors $A b$ and then stained with $1 \mu \mathrm{g}$ of b-Prl, anti-CD61-PE (BioLegend, San Diego, CA, USA), antiTER119-PE/Cy7 (BioLegend), anti-CD31-PE/Cy7 (BioLegend), anti-CD45-PE/Cy7 (BioLegend), anti-CD24-APC (BioLegend) and anti-CD49f-PerCP/Cy5.5 (BioLegend) for $30 \mathrm{~min}$ at $4{ }^{\circ} \mathrm{C}$. Cells were washed once in PBS $/ 1 \%$ BSA and incubated with Streptavidin-PE (SA-PE, eBioscience, San Diego, CA, USA) or Streptavidin-PETexas Red (SA-PE-TR, BD Biosciences) for $20 \mathrm{~min}$ on ice. For the analyses of nontransformed mammary glands, fat pads with no clinically apparent disease were examined. Inguinal mammary glands devoid of lymph nodes were harvested from mature 8-month-old WT or STAT1 ${ }^{-1-}$ retired breeders and processed as previously reported. ${ }^{18}$ Samples were collected using a LSRII (BD Bioscience). Gating procedure was described previously. ${ }^{18}$ Cells depleted of DAPI, CD31, CD45 and TER119 were analyzed based on CD49f and CD24 surface expression. Flow cytometry profiles were analyzed using FloJo software (TreeStar, Ashland, OR, USA). Myoepithelial cells were defined as CD49fi $C D 24^{\text {int }}$, whereas luminal epithelial cells were CD49fint $\mathrm{CD} 24^{\mathrm{hi}}$. Luminal epithelial cells or neoplastic cells were then gated on and analyzed for CD61 and PrIR levels. Apoptosis was measured by a flow cytometry-based annexin V-binding assay according to the manufacturer's instructions (BD Biosciences). Only early apoptotic cells (annexin V-positive, 7AAD-negative) were analyzed. Tyrosine phosphorylation of STAT1, STAT3 and STAT5A/5B was detected using antibodies specific for the phosphorylated forms of each STAT (anti-pSTAT1 from BD Biosciences; anti-pSTAT3 and anti-pSTAT5A/5B from Cell Signaling). Isotype control antibodies were used to set up the profile of the negative signal. Geometric mean channel shift is calculated as the difference between the geometric mean obtained from the PSTAT antibodies and that from the isotype control.

Production of anti-PrIR monoclonal antibody. As b-Prl bound PrIR at a lower affinity than PrIR-specific antibody, monoclonal antibody (MAb) against murine PrIR was produced. The extracellular domain (ECD) of murine PrIR (amino acids 25-239) was amplified from the cDNA of 129S6/SvEv MEC, ligated into the pEF4 vector (Invitrogen), and subsequently cloned into the pRK5 vector (a generous gift from J Darnell, Rockefeller University, New York, NY, USA). Recombinant PrlR-ECD was produced in Freestyle 293 Expression System (Invitrogen) and purified by using a Ni-NTA column (Qiagen, Valencia, CA, USA). Immunization of Armenian hamsters and production of MAb has been previously described. ${ }^{55}$ MAb clone $5 A 12$ is specific for murine PrIR (Supplementary Figures S4E and S4F).

Statistical analyses. Kaplan-Meier survival curve was analyzed by log-rank test. Tumor growth curve was analyzed by a distribution-free test. ${ }^{18}$ Repopulating capability of tumor cell sub-populations was analyzed by limiting dilution assay. ${ }^{56}$ All numerical results are presented as mean \pm S.E.M. and represent data from a minimum of three independent experiments unless otherwise stated. Unpaired $t$ test was used to determine the statistical significance between control and experimental groups. All tests are two-sided and a $P$-value $\leq 0.05$ was considered significant.

\section{Conflict of Interest}

TMM is an employee of Bristol-Myers Squibb. MVL is an employee of Johnson and Johnson. The remaining authors declare no conflict of interest.

Acknowledgements. We thank A Miceli, E Unanue and J Weber (Washington University) for critical review of the manuscript. We are also grateful to $G$ Longmore (Washington University) for the pFLRU lentivirus plasmids, J Darnell (Rockefeller University) for the pRK5 plasmid and L Lucini (University of Brescia) for analyzing human breast cancer samples. This work was supported by grants from the National Cancer Institute (RDS), the Ludwig Institute for Cancer Research (RDS) and Fondazione Beretta (WV). SL is supported by Borsa di studio Prof Roberto Tosoni.

1. EBCTCG. Effects of chemotherapy and hormonal therapy for early breast cancer on recurrence and 15-year survival: an overview of the randomised trials. Lancet 2005; 365 : 1687-1717.

2. Tworoger SS, Hankinson SE. Prolactin and breast cancer etiology: an epidemiologic perspective. J Mammary Gland Biol Neoplasia 2008; 13: 41-53.

3. Walker SR, Nelson EA, Zou L, Chaudhury M, Signoretti S, Richardson A et al. Reciprocal effects of STAT5 and STAT3 in breast cancer. Mol Cancer Res 2009; 7: 966-976.

4. Ormandy CJ, Hall RE, Manning DL, Robertson JF, Blamey RW, Kelly KA. Coexpression and cross-regulation of the prolactin receptor and sex steroid hormone receptors in breast cancer. J Clin Endocrinol Metab 1997; 82: 3692-3699. 
5. Touraine P, Martini JF, Zafrani B, Durand JC, Labaille F, Malet C et al. Increased expression of prolactin receptor gene assessed by quantitative polymerase chain reaction in human breast tumors versus normal breast tissues. J Clin Endocrinol Metab 1998; 83: 667-674.

6. Cotarla I, Ren S, Zhang Y, Gehan E, Singh B, Furth PA. Stat5a is tyrosine phosphorylated and nuclear localized in a high proportion of human breast cancers. Int J Cancer2004; 108: 665-671.

7. Arendt LM, Schuler LA. Transgenic models to study actions of prolactin in mammary neoplasia. J Mammary Gland Biol Neoplasia 2008; 13: 29-40.

8. lavnilovitch E, Cardiff RD, Groner B, Barash I. Deregulation of STAT5 expression and activation causes mammary tumors in transgenic mice. Int J Cancer 2004; 112: 607-619.

9. Bole-Feysot C, Goffin V, Edery M, Binart N, Kelly PA. Prolactin (PRL) and its receptor: actions, signal transduction pathways and phenotypes observed in $\mathrm{PRL}$ receptor knockout mice. Endocr Rev 1998; 19: 225-268.

10. Liu X, Robinson GW, Wagner K-U, Garrett L, Wynshaw-Boris A, Hennighausen L. Stat5a is mandatory for adult mammary gland development and lactogenesis. Genes Dev 1997; 11: 179-186.

11. Cui Y, Riedlinger G, Miyoshi K, Tang W, Li C, Deng C-X et al. Inactivation of STAT5 in mouse mammary epithelium during pregnancy reveals distinct functions in cell proliferation, survival, and differentiation. Mol Cell Biol 2004; 24: 8037-8047.

12. Teglund S, McKay C, Schuetz E, van Deursen JM, Stravopodis D, Wang D et al. Stat5a and Stat5b proteins have essential and nonessential, or redundant, rolds in cytokine responses. Cell 1998; 93: 841-850.

13. Wagner K-U, Krempler A, Triplett AA, Qi Y, George NM, Zhu J et al. Impaired alveologenesis and maintenance of secretory mammary epithelial cells in JAK2 conditional knockout mice. Mol Cell Biol 2004; 24: 5510-5520.

14. Han Y, Watling D, Rogers NC, Stark GR. JAK2 and STAT5, but not JAK1 and STAT1, are required for prolactin induced $\beta$-lactoglobulin transcription. Mol Endocrinol 1997; 11: 1180-1188.

15. David M, Petricoin EFr, Igarashi K, Feldman GM, Finbloom DS, Larner AC. Prolactin activates the interferon-regulated $\mathrm{p} 91$ transcription factor and the JAK2 kinase by tyrosine phosphorylation. Proc Natl Acad Sci USA 1994; 91: 7174-7178.

16. Gilmour KC, Reich NC. Receptor to nucleus signaling by prolactin and interleukin 2 via activation of latent DNA-binding factors. Proc Natl Acad Sci USA 1994; 91: 6850-6854.

17. DaSilva L, Rui H, Erwin RA, Howard OMZ, Kirken RA, Malabarba MG et al. Prolactin recruits STAT1, STAT3 and STAT5 independent of conserved receptor tyrosines TYR402, TYR479, TYR515 and TYR580. Mol Cell Endocrinol 1996; 117: 131-140.

18. Chan SR, Vermi W, Luo J, Lucini L, Rickert C, Fowler AM et al. STAT1-deficient mice spontaneously develop estrogen receptor a-positive luminal mammary carcinomas. Breast Cancer Res 2012; 14: R16.

19. Schneckenleithner C, Bago-Horvath Z, Dolznig H, Neugebauer N, Kollmann K, Kolbe T et al. Putting the brakes on mammary tumorigenesis: loss of STAT1 predisposes to intraepithelial neoplasias. Oncotarget 2011; 2: 1043-1054.

20. Klover PJ, Muller WJ, Robinson GW, Pfeiffer RM, Yamaji D, Hennighausen L. Loss of STAT1 from mouse mammary epithelium results in an increased Neu-induced tumor burden. Neoplasia 2010; 12: 899-905.

21. Raven JF, Williams V, Wang S, Tremblay ML, Muller WJ, Durbin JE et al. Stat1 is a suppressor of ErbB2/Neu-mediated cellular transformation and mouse mammary gland tumor formation. Cell Cycle 2011; 10: 794-804.

22. Purandare AV, McDevitt TM, Wan H, You D, Penhallow B, Han X et al. Characterization of BMS-911543, a functionally selective small-molecule inhibitor of JAK2. Leukemia 2012; 26: 280-288.

23. Yu H, Pardoll D, Jove R. STATs in cancer inflammation and immunity: a leading role for STAT3. Nat Rev Cancer 2009; 9: 798-809.

24. Garcia R, Bowman TL, Niu G, Yu H, Minton S, Muro-Cacho CA et al. Constitutive activation of STAT3 by the Src and JAK tyrosine kinases participates in growth regulation of human breast carcinoma cells. Oncogene 2001; 20: 2499-2513.

25. Leong PL, Andrews GA, Johnson DE, Dyer KF, Xi S, Mai JC et al. Targeted inhibition of STAT3 with a decoy oligonucleotide abrogates head and neck cancer cell growth. Proc Natl Acad Sci USA 2003; 100: 4138-4143.

26. Shackleton M, Vaillant F, Simpson KJ, Stingl J, Smyth GK, Asselin-Labat ML et al. Generation of a functional mammary gland from a single stem cell. Nature 2006; 439: 84-88.

27. Stingl J, Eirew P, Ricketson I, Shackleton M, Vaillant F, Choi D et al. Purification and unique properties of mammary epithelial stem cells. Nature 2006; 439: 993-997.

28. Van Keymeulen A, Rocha AS, Ousset M, Beck B, Bouvencourt G, Rock J et al. Distinct stem cells contribute to mammary gland development and maintenance. Nature 2011; 479: 189-193.

29. Tomic S, Chughtai N, Ali S. SOCS-1, -2, -3: selective targets and functions downstream of the prolactin receptor. Mol Cell Endocrinol 1999; 158: 45-54.

30. Helman D, Sandowski Y, Cohen Y, Matsumoto A, Yoshimura A, Merchav S et al. Cytokindinducible SH2 protein (CIS3) and JAK2 binding protein (JAB) abolish prolactin receptormediated STAT5 signaling. FEBS Lett 1998; 441: 287-291.
31. Pezet A, Favre H, Kelly PA, Edery M. Inhibition and restoration of prolactin signal transduction by suppressors of cytokind signaling. J Biol Chem 1999; 274: 24497-24502.

32. Ormandy CJ, Naylor M, Harris J, Robertson F, Horseman ND, Lindeman GJ et al. Investigation of the transcriptional changes underlying functional defects in the mammary glands of prolactin receptor knockout mice. Recent Prog Horm Res 2003; 58: 297-323.

33. Lindeman GJ, Wittlin S, Lada H, Naylor MJ, Santamaria M, Zhang J-G et al. SoCS1 deficiency results in accelerated mammary gland development and rescues lactation in prolactin receptor-deficient mice. Genes Dev 2001; 15: 1631-1636.

34. Harris J, Stanford PM, Sutherland K, Oakes SR, Naylor MJ, Robertson FG et al. Socs2 and Elf5 mediate prolactin-induced mammary gland development. Mol Endocrinol 2006; 20: 1177-1187.

35. Naka T, Fujimoto M, Tsutsui $H$, Yoshimura A. Negative regulation of cytokine and TLR signalings by SOCS and others. Adv Immunol 2005; 87: 61-122.

36. Sutherland KD, Lindeman GJ, Choong DY, Wittlin S, Brentzell L, Phillips W et al. Differential hypermethylation of SOCS genes in ovarian and breast carcinomas. Oncogene 2004; 23: 7726-7733.

37. Yamaji D, Na R, Feuermann Y, Pechhold S, Chen W, Robinson GW et al. Development of mammary luminal progenitor cells is controlled by the transcription factor STAT5A. Genes Dev 2009; 23: 2382-2387.

38. Rose-Hellekant TA, Arendt LM, Schroeder MD, Gilchrist K, Sandgren EP, Schuler LA. Prolactin induces $\mathrm{ER} \alpha$-positive and $\mathrm{ER} \alpha$-negative mammary cancer in transgenic mice. Oncogene 2003; 22: 4664-4674.

39. Sakamoto K, Triplett AA, Schuler LA, Wagner KU. Janus kinase 2 is required for the initiation but not maintenance of prolactin-induced mammary cancer. Oncogene 2010; 29: 5359-5369.

40. Frech MS, Halama ED, Tilli MT, Singh B, Gunther EJ, Chodosh LA et al. Deregulated estrogen receptor alpha expression in mammary epithelial cells of transgenic mice results in the development of ductal carcinoma in situ. Cancer Res 2005; 65: 681-685.

41. Caffarel MM, Zaragoza R, Pensa S, Li J, Green AR, Watson CJ. Constitutive activation of JAK2 in mammary epithelium elevates Stat5 signalling, promotes alveologenesis and resistance to cell death, and contributes to tumourigenesis. Cell Death Differ 2011; 19: 511-522.

42. Nevalainen MT, Xie J, Bubendorf L, Wagner KU, Rui H. Basal activation of transcription factor signal transducer and activator of transcription (Stat5) in nonpregnant mouse and human breast epithelium. Mol Endocrinol 2002; 16: 1108-1124.

43. Watson CJ, Neoh K. The Stat family of transcription factors have diverse roles in mammary gland development. Sem Cell Dev Biol 2008; 19: 401-406.

44. Liu X, Robinson GW, Hennighausen L. Activation of STAT5a and STAT5b by tyrosine phosphorylation is tightly linked to mammary gland differentiation. Mol Endocrinol 1996; 10: 1496-1506.

45. Letourneur M, Valentino L, Travagli-Gross J, Bertoglio J, Pierre J. Sp2 regulates interferongamma-mediated socs1 gene expression. Mol Immunol 2009; 46: 2151-2160.

46. Saito H, Morita Y, Fujimoto M, Narazaki M, Naka T, Kishimoto T. IFN regulatory factor-1mediated transcriptional activation of mouse STAT-induced STAT inhibitor-1 gene promoter by IFN-gamma. J Immunol 2000; 164: 5833-5843.

47. Clevenger CV, Zheng J, Jablonski EM, Galbaugh TL, Fang F. From bench to bedside: future potential for the translation of prolactin inhibitors as breast cancer therapeutics. J Mammary Gland Biol Neoplasia 2008; 13: 147-156.

48. Marotta LL, Almendro V, Marusyk A, Shipitsin M, Schemme J, Walker SR et al. The JAK2/ STAT3 signaling pathway is required for growth of CD44 CD24 stem cell-like breast cancer cells in human tumors. J Clin Invest 2011; 121: 2723-2735.

49. Hedvat M, Huszar D, Herrmann A, Gozgit JM, Schroeder A, Sheehy A et al. The JAK2 inhibitor AZD1480 potently blocks Stat3 signaling and oncogenesis in solid tumors. Cancer Cell 2009; 16: 487-497.

50. Britschgi A, Andraos R, Brinkhaus H, Klebba I, Romanet V, Muller U et al. JAK2/STAT5 inhibition circumvents resistance to $\mathrm{PI} 3 \mathrm{~K} / \mathrm{mTOR}$ blockade: a rationale for cotargeting these pathways in metastatic breast cancer. Cancer Cell 2012; 22: 796-811.

51. Weaver BK, Bohn E, Judd BA, Gil MP, Schreiber RD. ABIN-3: a molecular basis for species divergence in interleukin-10-induced anti-inflammatory actions. Mol Cell Biol 2007; 27: 4603-4616.

52. Horvath CM, Wen Z, Darnell JE Jr. A STAT protein domain that determines DNA sequence recognition suggests a novel DNA-binding domain. Genes Dev 1995; 9: 984-994.

53. Jin H, Lanning NJ, Carter-Su C. JAK2, but not Src family kinases, is required for STAT, ERK, and Akt signaling in response to growth hormone in preadipocytes and hepatoma cells. Mol Endocrinol 2008; 22: 1825-1841.

54. Dontu G, Abdallah WM, Foley JM, Jackson KW, Clarke MF, Kawamura MJ et al. In vitro propagation and transcriptional profiling of human mammary stem/progenitor cells. Genes Dev 2003; 17: 1253-1270.

55. Sheehan KCF, Schreiber RD. Generation and characterization of hamster monoclonal antibodies that neutralize murine necrosis factors. J. Immunol 1989; 142: 3884-3893.

56. Hu Y, Smyth GK. ELDA: extreme limiting dilution analysis for comparing depleted and enriched populations in stem cell and other assays. J Immunol Methods 2009; 347: 70-78. 